\title{
Opioid epidemic-- is there an end in sight?
}

Sean Loudin $\mathrm{MD}^{1}$, Mehiar El-Hamdani $\mathrm{MD}^{1}$, Mohammed Ranavaya MD, JD, MS, FRCPI, FFOM, CIME ${ }^{1}$

\section{Author Affiliations:}

1. Marshall University Joan C. Edwards School of Medicine, Huntington, West Virginia

\section{Corresponding Author:}

Mohammed Ranavaya MD, JD, MS, FFOM, FRCPI

Marshall University Joan C. Edwards School of Medicine

Huntington, West Virginia

Email: ranavaya@marshall.edu 
According to CDC more than 165 people die every day in the US of a drug overdose. This amounts to fatalities in a jumbo jet crash every other day. Two significant factors fuel this alarming trend - the misuse of prescription drugs and a related increase in heroin use. The agency for healthcare research and quality (AHRQ) data shows that in the last decade there was $99 \%$ increase in emergency room visits and about $66 \%$ inpatient increase for opioid related problems. Calling it an epidemic is stating the obvious. This epidemic results in an enormous cost to the person, families and society.

The problem of addiction spares no age and afflicts even the unborn. Neonatal Abstinence Syndrome (NAS) occurs when a fetus has been exposed to neuroactive substances in utero and then experiences withdrawal symptoms after birth. WV leads the nation in the incidence of NAS. In WV approximately 58/1000 (5.8\%) live births are diagnosed with NAS and about 140/1000 (14\%) live births are exposed to substances in utero. These numbers reflect that WV deals with NAS and maternal substance exposure at a rate eight to nine times higher than that of the rest of the country. NAS is just the tip of the iceberg. NAS is the end result and a small representation of the grand scope of substance use disorder ravaging our community. Identifying the various substances these infants are exposed to reflect the ever changing face of substance abuse. Women of childbearing age are a subset of the entire community grappling with substance use disorder. When they become pregnant their child will be at risk for developing withdrawal. When an infant is born exposed to drugs in utero a referral to Child Protective Services (CPS) must be made by the hospital to comply with federal regulations. CPS must then evaluate the safety of each individual's circumstances to prepare for the best outcome upon discharge.

Ideally there are supportive circumstances in the home: mother and/or father in recovery or stable in a supervised treatment program, family support in a substance free environment, social services and early intervention programs available to those at risk families, and motivation and assistance to remain compliant with treatment. Unfortunately those circumstances are not always able to be achieved; so infants must have alternative placement or worse outcomes if the system fails them and they are sent home into a dangerous environment. Our area is at the heart of the opioid epidemic but it has also seen the rise of public health emergencies as a result of substance abuse. The growing rates of hepatitis $\mathrm{C}$ are alarming. Harm reduction strategies are being implemented across the state to reduce the rates of hepatitis $\mathrm{C}$. The consequence of adults and women of childbearing age with hepatitis $\mathrm{C}$ are infants born to hepatitis $\mathrm{C}$ positive mothers. The long term impact on these children can potentially be catastrophic. The vertical transmission rate may be on the order of five to eight percent. Identifying children with hepatitis $\mathrm{C}$ relies on antibody testing at around eighteen months of age. Missing this diagnosis can lead to a variety of health complications throughout adolescence and adulthood.

The response to current opioid epidemic needs to be multipronged. Education about substance abuse must start very early. Lack of education is a barrier for all of us. Education about substance use disorder is necessary for all walks of life and all professions: teachers, social workers, doctors, nurses, judges, lawyers, police officers, first responders, pastors, priests, bankers, etc. Tackling any problem first requires knowledge about the causes, current status, future directions, and passion for change. 
Effective behavioral therapy must be implemented for individuals struggling with substance use disorder. Behavioral health professionals dedicated to substance use disorder are lacking across the nation, but WV certainly lacks providers and has such a large number of patients affected by substance use disorder that current providers are overwhelmed. Early intervention programs for infants and families affected by substance use disorder are stretched thin. Support for the WV DHHR (Department of Health and Human Resources) and CPS is critical because they need consistency and the ability to make sound safety decisions that are in the child's best interest. Funding for innovative programs making a difference in the community (Lily's Place, first responder programs, harm reduction clinics, etc) is essential. Economic revitalization within a community could provide individuals with purpose and motivation instead of hopelessness and despair. Increasing the workforce could also allow for money to flow back into the community thus assisting in overall recovery for the community as a whole.

Responsible prescribing practices with opiates for pain but also responsible clinicians running MAT (Medication Assisted Therapy) programs must be achieved. Economic stability and growth must provide motivation and hope to individuals looking for a way to support themselves and their families. The judicial system must recognize the cycle of recovery, that there needs to be support, that relapse is a portion of recovery, and that we cannot incarcerate our way out of this problem. Government needs to be able to support programs that are effective in responding to this crisis. There should be funding for successful endeavors and the ability to trim funding to programs or institutions that aren't doing the right thing.

The current addiction epidemic is a perfect storm of other societal issues that we have left unaddressed in United States. The increase in opiate abuse in United States as well as its related death rates correlates quite well with increased feelings of isolation amongst teens as well as increased desire to pursue risky behavior amongst our youth populations. Therefore, any longterm solution to the opioid crisis must involve addressing the issues plaguing the psychological health of our society. It is not enough to merely legislate and enforce laws that ultimately deal with the epidemic after-the-fact without simultaneously addressing the issues that have created the problem. But that being said, there has to be an active societal effort particularly at the school level focused on reducing those external factors shown to increase isolation and depression in our youth such as excessive use of social media. Furthermore, there needs to be more active effort at curbing risky behavior in general in our youth population through school programs and other social programs. I believe it is those initial behaviors that then allow for the pursuance of the more deviant behaviors in a later time. The existing school programs currently focus on the risks of tobacco use but only gloss over the risks of opioid use and abuse, and in expanding the school programs, instructors should widen the field of concern to include this class of drugs.

Obviously, these types of mass social analysis and intervention will require major financial support and manpower. Improving the economic situation in Huntington and surrounding areas is pivotal. By focusing on improving the economic aspects of the town we will raise the quality of life for the residents, increase resources and their accessibility, which in turn can reshape the existing culture. Huntington and surrounding areas need to be extremely active to raise funds and investors need to be encouraged to help improve the status of the community. The barriers involved with implementing this solution involve the state of mind of the community. This state of mind has to be actively changed by community leaders from Marshall University, the medical 
school, local businesses, and by key politicians and other prominent figures. All have to come together for one cause. If the community does not believe it has a chance to improve, then no one will put time and effort, donate, or invest in the community. If the community has hope in its recovery then people will act aggressively and positively because they will be more confident that they are working towards a better future for themselves and society. 